\title{
Subcortical and Deep Cortical Atrophy in Frontotemporal Dementia due to Granulin Mutations
}

\author{
Enrico Premi ${ }^{a}$ Valentina Garibotto ${ }^{d}$ Stefano Gazzina ${ }^{a}$ Anna Formenti ${ }^{a}$ \\ Silvana Archettic ${ }^{c}$ Roberto Gasparotti ${ }^{b}$ Alessandro Padovani ${ }^{a}$ \\ Barbara Borroni ${ }^{a}$ \\ ${ }^{a}$ Centre for Ageing Brain and Neurodegenerative Disorders, Neurology Unit, and \\ ${ }^{b}$ Neuroradiology Unit, University of Brescia, and ' III Laboratory of Analysis, Brescia Hospital, \\ Brescia, Italy; ${ }^{d}$ Department of Medical Imaging, Geneva University Hospital, \\ Geneva, Switzerland
}

\section{Key Words}

Frontotemporal dementia - Granulin - Magnetic resonance imaging - Subcortical regions .

Basal ganglia · Probabilistic atlases $\cdot$ Voxel-based morphometry

\section{Abstract}

Background/Aims: Parkinsonism is often associated with symptoms of frontotemporal dementia (FTD), but its pathogenesis has been largely neglected. In genetic inherited FTD-related granulin (GRN) mutations, parkinsonism is an early sign, and it is more common than in sporadic disorders. Our aim was to study grey matter (GM) volume changes in subcortical and deep cortical regions in GRN-related FTD. Methods: A total of 33 FTD patients (13 carriers of the $G R N$ mutation, $G R N+$, and 20 non-carriers, $G R N-$ ) and 12 healthy controls ( $\mathrm{HC}$ ) were included in the study. Each subject underwent an MRI examination (1) for voxel-based morphometry to study GM differences in cortical and subcortical regions, and (2) for a region of interest approach using a probabilistic atlas of subcortical regions (caudate nucleus, putamen, thalamus and amygdala) to assess the regional differences. Results: The GRN+ group showed greater damage in frontotemporal regions than the GRN-group. The FTD patients had greater GM atrophy in the caudate nucleus and in the thalamus bilaterally than the HC. Damage to these subcortical and deep cortical regions was greater in the GRN+ than in the GRN-patients. Discussion: Subcortical and deep cortical involvement is a key feature of FTD, and more pronounced in $G R N$-related disease. Damage to the caudate region in $G R N+$ patients may explain the parkinsonism frequently associated since the early stages of the disease. 

Premi et al.: Subcortical and Deep Cortical Atrophy in Frontotemporal Dementia due to
Granulin Mutations

\section{Introduction}

Frontotemporal dementia (FTD) is a neurodegenerative disorder characterized by behavioural abnormalities, language impairment and deficits of executive functions [1,2]. A number of disease-causative genes have been identified so far [3]. Loss-of-function mutations within the granulin (GRN) gene represent one of the best-recognized pathogenetic determinants along with mutations within MAPT and C9orf72 [4]. Patients carrying GRN mutations usually show variable age at onset and clinical presentation, ranging from behaviouralvariant FTD to prevalent involvement of language functions, i.e. agrammatic-variant primary progressive aphasia, or corticobasal syndrome [5]. In all these phenotypes, parkinsonism is present early during the course of the disease. Histopathological studies confirmed the presence of subcortical atrophy in FTD patients, involving the caudate nucleus, putamen, globus pallidus and amygdala [6-8]. Pathological studies as well as radiological analysis by MRI demonstrated a variable degree of atrophy in subcortical structures $[9,10]$ in $G R N$ related FTD, with a prevalent involvement of the caudate nucleus [9]. In this sense, starting from this earlier work [11], we performed a fully automated volumetric analysis using regions of interest (ROIs) based on probabilistic atlases $[12,13]$ even on $G R N$-related disease. These atlases are based on the anatomical examination of large samples of healthy subjects and provide information about the voxel-specific probability of observing a given structure in stereotactic space $[14,15]$. Such a methodological approach allowed a more precise definition of subcortical regional atrophy, improving the accuracy of the analysis, compared with voxelwise statistical approaches. In fact, global statistical significance in a specific anatomical structure can be reached even if it is absent at the level of single voxels [11]. To this purpose, we firstly performed a voxelwise whole-brain analysis to assess the significant differences in grey matter (GM) density in the three groups of subjects, i.e. $G R N$-related FTD $(G R N+$ ) patients, non-GRN-related FTD (GRN-) patients and age-matched healthy controls (HC). After this, we measured the GM volumes of the caudate nucleus, putamen and thalamus as well as of the amygdala in order to define GM volume changes in relation to the clinical diagnosis (FTD) and the presence of mutation (GRN).

\section{Subjects and Methods}

\section{Subjects}

The studied sample included 33 patients with FTD, all genetically characterized by the presence/absence of GRN and MAPT mutations and C9orf72 hexanucleotide expansion. Twelve of them resulted to be carriers of the GRN Thr272fs mutation (GRN+), while 20 were non-carriers of the genetic variations screened for (GRN-). Twelve age- and gender-matched HC were enrolled as a control group. All patients met the current clinical diagnostic criteria for FTD $[1,2]$. To increase as much as possible our confidence in the correctness of the diagnosis of FTD patients without GRN Thr272fs mutation, they had to have been clinically and neuropsychologically followed up for at least 2 years by the time of recruitment, and the diagnosis had to be confirmed.

All patients underwent a clinical and neurological evaluation, a routine laboratory examination and brain MRI. An extensive neuropsychological assessment, including the FTDmodified Clinical Dementia Rating scale, was conducted, as previously described [16].

Written informed consent (from the subject, or from the responsible guardian if the subject was incapable) was obtained before study initiation for each procedure, including blood collection from venous puncture, genetic analysis and MRI. The work conformed to the Declaration of Helsinki and was approved by the local ethics committee. 


\section{GRN Sequencing}

Genomic DNA was extracted from peripheral blood using a standard procedure. All the 12 exons plus exon 0 of $G R N$ and at least $30 \mathrm{bp}$ of their flanking introns were evaluated by polymerase chain reaction and subsequent sequencing. The GRN Thr272fs (g.1977_1980 delCACT) mutation was tested for, as previously described $[17,18]$.

\section{Statistics for Demographic and Clinical Data}

The SPSS package (version 17.0; SPSS Inc., Chicago, Ill., USA) was used to run statistics for group differences in demographic and clinical characteristics. Group comparisons were made by the Mann-Whitney $U$ test or $\chi^{2}$ test, setting the statistical threshold at $p \leq 0.05$ (Bonferroni corrected).

\section{MRI Acquisition}

All images were obtained using a 1.5-tesla MR scanner (Siemens Symphony; Siemens, Erlangen, Germany) equipped with a circularly polarized transmit/receive coil. In a single session for each subject, a 3D MPRAGE (magnetization-prepared rapid acquisition gradient echo) T1-weighted scan was collected (TR $=2,010 \mathrm{~ms}$; TE $=3.93 \mathrm{~ms}$; matrix $=1 \times 1 \times 1$; in-plane field of view $=250 \times 250 \mathrm{~mm}$; slice thickness $=1 \mathrm{~mm}$; flip angle $=15^{\circ}$ ). Preprocessing and statistical analysis were performed using the SPM8 package (Wellcome Department of Imaging Neuroscience, London, UK; http://www.fil.ion.ucl.ac.uk/spm/) running on Matlab 7.6 (MathWorks, Natick, Mass., USA). T1-weighted images of all recruited subjects were visually inspected for qualitative assessment of macroscopic atrophy and to check the quality of the data before carrying out a quantitative volumetric analysis. The MPRAGE data were processed using the voxel-based morphometry protocol in SPM8. For each subject, an iterative combination of segmentations and normalizations (implemented within the 'segment' module) produced a GM probability map [19] in Montreal Neurological Institute coordinates. To compensate for compression or expansion during warping of images to match the template, the GM maps were modulated by multiplying the intensity of each voxel by the local value derived from the deformation field (Jacobian determinants) [20]. All data were then smoothed using a $10-\mathrm{mm}$ full width at half maximum Gaussian kernel. For the voxelwise analysis, a three-group ANOVA statistical design was defined. The statistical threshold was set at $\mathrm{p}<$ 0.05 , familywise error (FWE) corrected, for the comparisons between patients and HC (GRN+ vs. HC and GRN- vs. HC). For the direct comparison between the two groups of patients (GRN+ vs. GRN-), a less stringent statistical threshold was set at $\mathrm{p}<0.01$ (uncorrected), and only the clusters surviving at FWE 0.05 (FWE 0.05 cluster level) were considered, as previously described [21].

ROIs were derived using a probabilistic map-based approach, as previously described and validated [11]. For each voxel of the reference space, probabilistic maps described the frequency with which a given area was observed at this specific position in a reference sample, i.e. how likely it was to be found at a given position. We used two sets of probabilistic maps: one for the caudate nucleus, putamen and thalamus, derived from the International Consortium for Brain Mapping atlas (http://www.loni.ucla.edu/atlases), and one for the amygdala, based on cytoarchitectonic examination, as described in Amunts et al. [22]. From these probabilistic maps we computed a maximum probability map (MPM), allowing us to classify voxels into distinct and non-overlapping ROIs for each area. The MPM attributes each voxel to a specific, most likely region by comparing the probabilities for all areas observed at any given position in a population [12]. Thus, the visualization and use of MPMs are similar to those of conventional brain atlases, but MPMs represent the most likely area at each position rather than a single 'typical' hemisphere. The extracted GM volumes were subsequently corrected for total intracranial volume. We previously provided a validation of this set of ROIs for the assessment 
Premi et al:: Subcortical and Deep Cortical Atrophy in Frontotemporal Dementia due to Granulin Mutations

Table 1. Demographic and clinical characteristics of FTD subjects, sorted by GRN mutation

\begin{tabular}{|c|c|c|c|c|}
\hline & $\begin{array}{l}\text { FTD overall } \\
(\mathrm{n}=33)\end{array}$ & $\begin{array}{l}\text { FTD-GRN+ } \\
(\mathrm{n}=13)\end{array}$ & $\begin{array}{l}\text { FTD-GRN- } \\
(\mathrm{n}=20)\end{array}$ & $\mathrm{p}^{\mathrm{a}}$ \\
\hline Age at evaluation, years & $65.6 \pm 7.0$ & $62.9 \pm 5.9$ & $67.4 \pm 7.2$ & 0.069 \\
\hline Male gender, n (\%) & $57.6(19)$ & $30.8(4)$ & $75.0(15)$ & $0.030^{\mathrm{b}}$ \\
\hline Age at onset, years & $62.0 \pm 6.8$ & $59.0 \pm 6.1$ & $64.0 \pm 6.6$ & $0.035^{\mathrm{b}}$ \\
\hline Disease duration, years & $3.0 \pm 2.4$ & $2.4 \pm 2.0$ & $3.4 \pm 2.7$ & 0.272 \\
\hline Education, years & $7.2 \pm 2.6$ & $7.2 \pm 2.8$ & $7.4 \pm 2.6$ & 0.904 \\
\hline Family history, n (\%) & $63.6(21)$ & $100.0(13)$ & $40.0(8)$ & $0.001^{\mathrm{b}}$ \\
\hline MMSE score & $21.6 \pm 7.6$ & $18.9 \pm 9.3$ & $23.3 \pm 5.9$ & 0.153 \\
\hline FTD-modified CDR & $5.9 \pm 4.8$ & $8.0 \pm 6.2$ & $4.5 \pm 3.1$ & 0.081 \\
\hline bvFTD phenotype, n (\%) & $54.5(18)$ & $46.2(6)$ & $60.0(12)$ & 0.493 \\
\hline NPI score & $14.2 \pm 9.3$ & $18.2 \pm 11.6$ & $11.7 \pm 6.6$ & 0.083 \\
\hline FBI-A score & $10.0 \pm 6.7$ & $12.7 \pm 7.2$ & $8.3 \pm 6.0$ & 0.077 \\
\hline FBI-B score & $4.0 \pm 4.0$ & $4.9 \pm 5.1$ & $3.5 \pm 3.2$ & 0.407 \\
\hline FBI-AB score & $14.0 \pm 9.1$ & $17.5 \pm 10.0$ & $11.8 \pm 7.9$ & 0.091 \\
\hline UPDRS-III score & $4.7 \pm 5.6$ & $4.3 \pm 4.3$ & $5.1 \pm 6.8$ & 0.784 \\
\hline
\end{tabular}

Values denote means \pm SD unless specified otherwise. MMSE = Mini-Mental State Examination; FTD-modified CDR = frontotemporal dementia-modified clinical dementia rating scale; bvFTD = behaviouralvariant FTD; NPI = Neuropsychiatric Inventory; FBI = Frontal Behavioural Inventory; UPDRS = Unified Parkinson's Disease Rating Scale.

${ }^{\mathrm{a}} \mathrm{t}$ test, otherwise specified. ${ }^{\mathrm{b}} \chi^{2}$ test (comparing FTD-GRN+ with FTD-GRN-).

of basal ganglion and amygdala volumes in a population of patients with frontotemporal lobar degeneration [11]. GM volumes for each area from each subject were then compared across groups by a three-way ANOVA design, and post hoc comparisons were done by pairwise $t$ tests, using the Bonferroni correction for multiple comparisons $(\mathrm{p}<0.05)$. The analyses were performed using the SPSS software package (version 16.0 for Windows; SPSS Inc.).

\section{Results}

\section{Subjects}

The demographic and clinical characteristics of the $13 G R N+$ and the $20 G R N$ - subjects are shown in table 1 . The $G R N+$ group showed, as expected, a higher prevalence of a positive family history as well as a significantly earlier onset of the disease. The two groups were comparable regarding demographic characteristics, disease duration and disease severity, as measured by the Clinical Dementia Rating scale modified for FTD, and behavioural disturbances, as measured by the Frontal Behavioural Inventory and Neuropsychiatric Inventory.

\section{Voxelwise Analysis}

Overall, the FTD patients showed significant bilateral GM atrophy in frontotemporal regions compared with the $\mathrm{HC}$ (fig. 1a). The $G R N+$ subjects presented greater frontotemporal damage than the GRN- subjects (fig. 1b, c). Furthermore, as shown in figure 2, in the GRN+ patients there was more severe involvement of these regions than in the $G R N$-patients (x, y, $\mathrm{z}:-6,-18,-8$ left thalamus, $\mathrm{T}=7.92$, cluster $=37,860$, FWE cluster level $\mathrm{p}<0.001 ; \mathrm{x}, \mathrm{y}, \mathrm{z}: 8$, $-20,8$ right thalamus, $\mathrm{T}=6.08$, cluster $=37,860$, FWE cluster level $\mathrm{p}<0.001 ; \mathrm{x}, \mathrm{y}, \mathrm{z}:-34,-16$, -4 left inferior frontal gyrus, $\mathrm{T}=5.66$, cluster $=37,860$, FWE cluster level $\mathrm{p}<0.001$ ). 
Premi et al.: Subcortical and Deep Cortical Atrophy in Frontotemporal Dementia due to Granulin Mutations
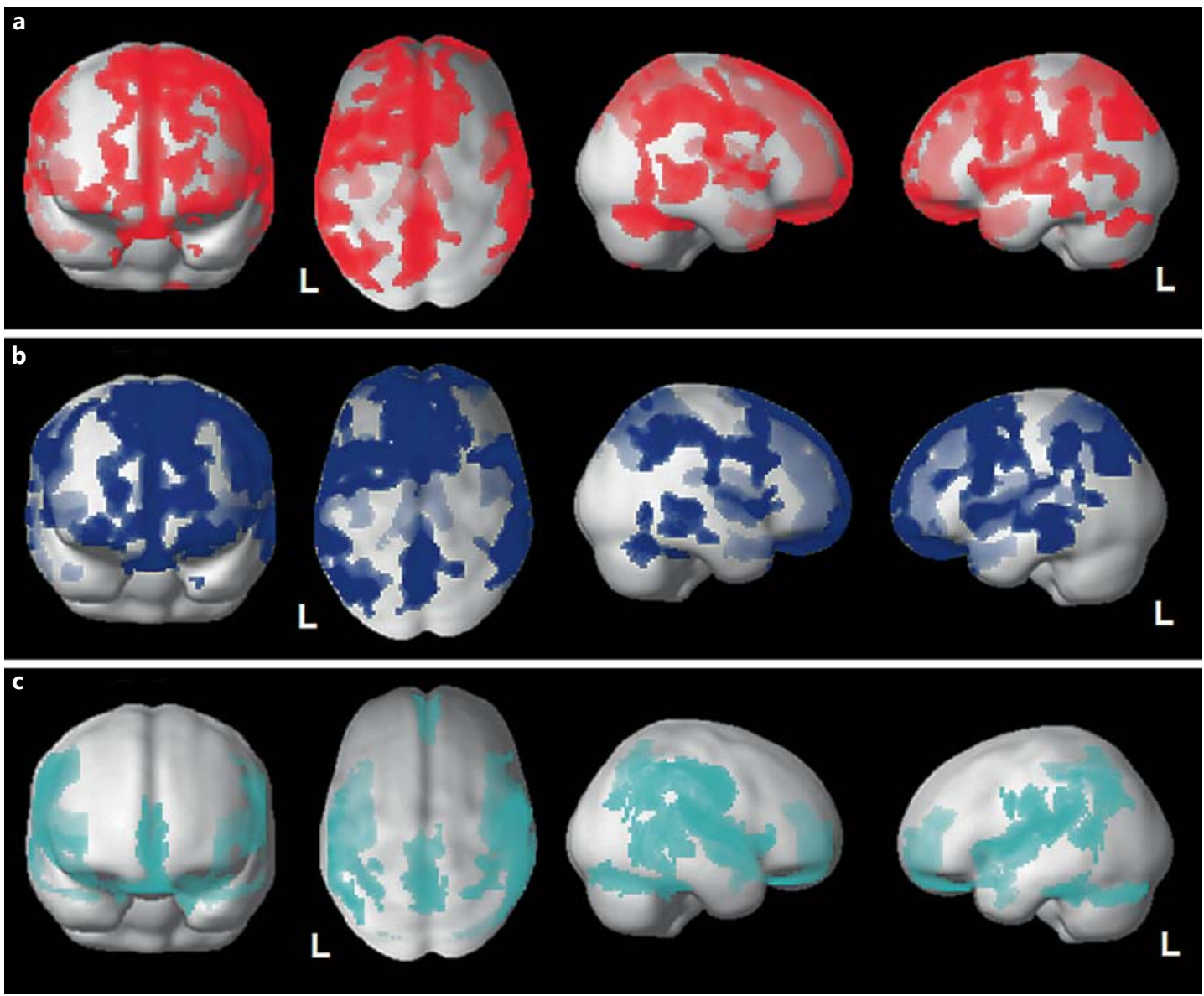

Fig. 1. a Voxelwise voxel-based morphometry analysis of all FTD patients (GRN+ and $G R N-$ ) compared with HC. b Voxelwise voxel-based morphometry analysis of $G R N+$ patients compared with HC. c Voxelwise voxelbased morphometry analysis of GRN-patients compared with HC. a-c All analyses are superimposed on a 3D normalized template. $\mathrm{p}<0.05$, FWE corrected. Voxel threshold: 200 voxels. $\mathrm{L}=\mathrm{Left}$.

\section{ROIs: Group Comparisons}

In table 2, the mean values for the ROIs in each of the areas evaluated are shown. Greater bilateral GM atrophy in the caudate nucleus and thalamus was observed in the FTD patients, and a main effect was demonstrated for the GRN+ group as compared with the GRN-group.

\section{Discussion}

In this work we studied the involvement of subcortical structures in $G R N$-related pathology using in vivo volumetric analysis, as previously described for sporadic patients with FTD [11]. First of all, in line with our previous findings [11], we confirmed the subcortical involvement in FTD. Furthermore, when considering the presence of $G R N$-related pathology, we found a significantly higher grade of atrophy in the caudate nucleus and thalamus bilaterally compared with $G R N$ - patients. The results of the ROI approach were also confirmed by voxelwise voxel-based morphometry analysis, with greater GM atrophy in the GRN+ group, especially in subcortical regions. The greater subcortical atrophy was not related to a more advanced clinical stage of 
Premi et al.: Subcortical and Deep Cortical Atrophy in Frontotemporal Dementia due to Granulin Mutations
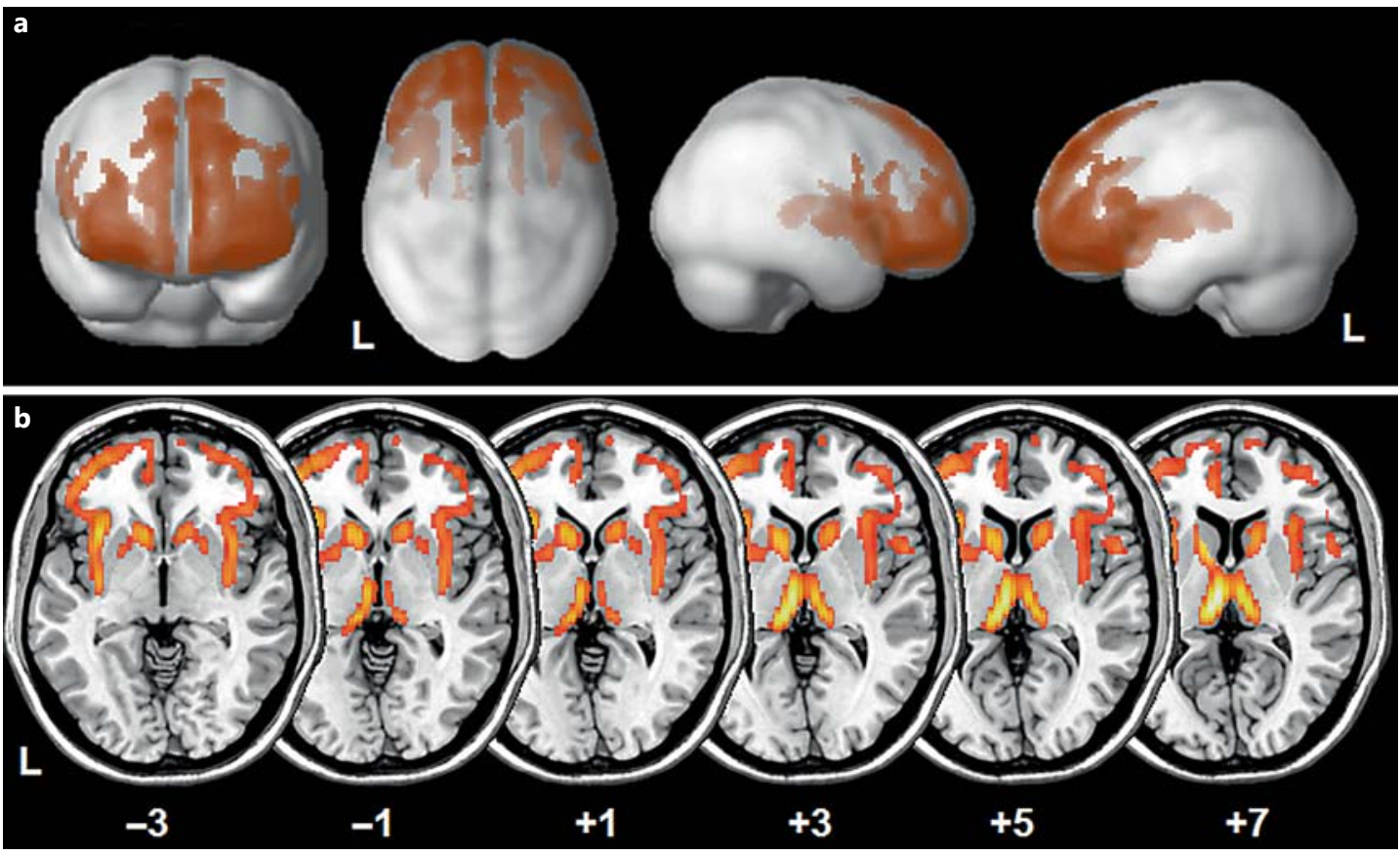

Fig. 2. a Voxelwise voxel-based morphometry analysis between $G R N+$ and $G R N-$ patients (GRN+< $G R N-$ ) superimposed on a 3D normalized template. b Voxelwise voxel-based morphometry analysis between $G R N+$ and $G R N$ - patients ( $G R N+<G R N-$ ) superimposed on a standardized axial MRI template at the level of subcortical regions to emphasize the greater subcortical involvement in $G R N+$ compared with $G R N-$ patients. The numbers denote Montreal Neurological Institute coordinates on the z-axis. $\mathbf{a}, \mathbf{b}$ p $<0.01$, uncorrected; only clusters surviving at the FWE-corrected 0.05 cluster level were considered. Voxel threshold: 200 voxels. $L=$ Left.

Table 2. GM volumes (divided by individual total intracranial volume) for each region and group (FTD-GRN+ and FTD-GRN-) compared with HC

\begin{tabular}{lllll}
\hline & FTD-GRN+ & FTD-GRN- & HC & p (ANOVA) \\
\hline Total GM volume & $0.495 \pm 0.460$ & $0.565 \pm 0.084$ & $0.620 \pm 0.057$ & $<0.001$ \\
L caudate nucleus & $1.104 \pm 0.768$ & $1.863 \pm 0.506$ & $2.514 \pm 0.398$ & $<0.001$ \\
R caudate nucleus & $1.152 \pm 0.978$ & $1.857 \pm 0.485$ & $2.533 \pm 0.415$ & $<0.001$ \\
L putamen & $1.930 \pm 0.404$ & $2.118 \pm 0.396$ & $2.039 \pm 0.294$ & n.s. \\
R putamen & $1.433 \pm 0.399$ & $1.739 \pm 0.426$ & $1.639 \pm 0.263$ & n.s. \\
L thalamus & $1.658 \pm 0.342$ & $2.065 \pm 0.310$ & $2.304 \pm 0.343$ & $<0.001$ \\
R thalamus & $1.726 \pm 0.451$ & $2.062 \pm 0.297$ & $2.291 \pm 0.302$ & 0.001 \\
L amygdala & $2.192 \pm 0.365$ & $2.139 \pm 0.290$ & $2.323 \pm 0.235$ & n.s. \\
R amygdala & $2.110 \pm 0.312$ & $2.150 \pm 0.298$ & $2.168 \pm 0.207$ & n.s. \\
\hline
\end{tabular}

Values correspond to regional and global GM value divided by total intracranial volume and multiplied by 100. Numbers are rounded to 3 significant figures. $\mathrm{L}=$ Left; $\mathrm{R}=$ right; $\mathrm{n} . \mathrm{s}$. = not significant.

${ }^{a}$ Comparisons (FTD-GRN+ with FTD-GRN-) surviving Bonferroni correction, $\mathrm{p}<0.05$, italicized. 
Premi et al.: Subcortical and Deep Cortical Atrophy in Frontotemporal Dementia due to Granulin Mutations

the disease in the GRN+ patients. Although the involvement of these subcortical structures (especially the caudate nucleus and thalamus) cannot be considered to be specific to $G R N$ related pathology (being evident also in the sporadic form), this is the first study that demonstrates a higher rate of GM atrophy in subcortical structures for GRN-related pathology, supporting its role in the complex networks that sustain the phenotypic presentation of the disease, such as extrapyramidal symptoms, frequently associated with behavioural and language impairments [10,23, 24]. As supported by histopathological studies [25-27], patients with GRN mutations present a highly consistent pattern characterized by more severe atrophy in the frontal lobe, severe involvement of the caudate nucleus as well as loss of pigmentation of the substantia nigra [28]. Furthermore, ubiquitin immunohistochemistry studies demonstrated that key elements of ubiquitinated frontotemporal lobar degeneration, such as neuronal cytoplasmic inclusions and lentiform ubiquitin-immunoreactive neuronal intranuclear inclusions, are highly represented in subcortical regions, especially the caudate nucleus and putamen [10].

From this perspective, the involvement of subcortical regions (like the caudate nucleus) and the substantia nigra could be the neuropathological basis for the parkinsonism commonly associated with the clinical course of $G R N$-related pathology [10]. Early involvement of these subcortical and deep cortical brain regions, even though not pathognomonic, may be considered a signature of $G R N$-related disease. GRN-related FTD encompasses a heterogeneous spectrum of clinical presentation related to frontotemporal and even parietal damage [29-31] compared with other FTD disorders. This study suggests that subcortical and deep cortical damage involves extra brain areas in GRN+ patients, thus further establishing the picture of the disease. The relationship between progranulin haploinsufficiency due to GRN mutations and the wide cerebral involvement of cortical, subcortical and deep cortical brain areas is still undetermined. However, this study suggests that $G R N$-related FTD is a more complex disease than was thought at the beginning, not only affecting frontal and temporal lobes. Further confirmatory research is warranted.

\section{Acknowledgements}

The authors wish to thank the patients and their families for taking part in this study.

\section{References}

1 Rascovsky K, Hodges JR, Knopman D, et al: Sensitivity of revised diagnostic criteria for the behavioural variant of frontotemporal dementia. Brain 2011;134:2456-2477.

-2 Gorno-Tempini ML, Hillis AE, Weintraub S, et al: Classification of primary progressive aphasia and its variants. Neurology 2011;76:1006-1014.

-3 Sieben A, van Langenhove T, Engelborghs S, Martin JJ, Boon P, Cras P, de Deyn PP, Santens P, van Broeckhoven C, Cruts M: The genetics and neuropathology of frontotemporal lobar degeneration. Acta Neuropathol 2012; 124:353-372.

-4 Rademakers R, Neumann M, Mackenzie IR: Advances in understanding the molecular basis of frontotemporal dementia. Nat Rev Neurol 2012;8:423-434.

5 van Swieten JC, Heutink P: Mutations in progranulin (GRN) within the spectrum of clinical and pathological phenotypes of frontotemporal dementia. Lancet Neurol 2008;7:965-974.

6 Hodges JR, Miller B: The classification, genetics and neuropathology of frontotemporal dementia: introduction to the special topic papers. Part I. Neurocase 2001;7:31-35.

7 Munoz DG, Dickson DW, Bergeron C, Mackenzie IR, Delacourte A, Zhukareva V: The neuropathology and biochemistry of frontotemporal dementia. Ann Neurol 2003;54(suppl 5):S24-S28.

-8 Yancopoulou D, Xuereb JH, Crowther RA, Hodges JR, Spillantini MG: Tau and $\alpha$-synuclein inclusions in a case of familial frontotemporal dementia and progressive aphasia. J Neuropathol Exp Neurol 2005;64:245-253.

-9 Mackenzie IR, Baker M, Pickering-Brown S, Hsiung GY, Lindholm C, Dwosh E, Gass J, Cannon A, Rademakers $\mathrm{R}$, Hutton M, Feldman HH: The neuropathology of frontotemporal lobar degeneration caused by mutations in the progranulin gene. Brain 2006;129:3081-3090. 
Premi et al.: Subcortical and Deep Cortical Atrophy in Frontotemporal Dementia due to Granulin Mutations

10 Josephs KA, Ahmed Z, Katsuse 0, et al: Neuropathologic features of frontotemporal lobar degeneration with ubiquitin-positive inclusions with progranulin gene (PGRN) mutations. J Neuropathol Exp Neurol 2007;66: 142-151.

11 Garibotto V, Borroni B, Agosti C, Premi E, Alberici A, Eickhoff SB, Brambati SM, Bellelli G, Gasparotti R, Perani D, Padovani A: Subcortical and deep cortical atrophy in frontotemporal lobar degeneration. Neurobiol Aging 2011;32:875-884.

12 Eickhoff SB, Stephan KE, Mohlberg H, Grefkes C, Fink GR, Amunts K, Zilles K: A new SPM toolbox for combining probabilistic cytoarchitectonic maps and functional imaging data. Neuroimage 2005;25:1325-1335.

13 Mazziotta J, Toga A, Evans A, et al: A probabilistic atlas and reference system for the human brain: International Consortium for Brain Mapping (ICBM). Philos Trans R Soc Lond B Biol Sci 2001;356:1293-1322.

14 Eickhoff SB, Heim S, Zilles K, Amunts K: Testing anatomically specified hypotheses in functional imaging using cytoarchitectonic maps. Neuroimage 2006;32:570-582.

15 Hammers A, Allom R, Koepp MJ, Free SL, Myers R, Lemieux L, Mitchell TN, Brooks DJ, Duncan JS: Three-dimensional maximum probability atlas of the human brain, with particular reference to the temporal lobe. Hum Brain Mapp 2003;19:224-247.

-16 Borroni B, Agosti C, Premi E, Cerini C, Cosseddu M, Paghera B, Bellelli G, Padovani A: The FTLD-modified Clinical Dementia Rating scale is a reliable tool for defining disease severity in frontotemporal lobar degeneration: evidence from a brain SPECT study. Eur J Neurol 2010;17:703-707.

-17 Borroni B, Alberici A, Premi E, Archetti S, Garibotto V, Agosti C, Gasparotti R, di Luca M, Perani D, Padovani A: Brain magnetic resonance imaging structural changes in a pedigree of asymptomatic progranulin mutation carriers. Rejuvenation Res 2008;11:585-595.

18 Borroni B, Bonvicini C, Galimberti D, et al: Founder effect and estimation of the age of the Progranulin Thr272fs mutation in 14 Italian pedigrees with frontotemporal lobar degeneration. Neurobiol Aging 2011;32:555e1e8.

19 Ashburner J, Friston KJ: Unified segmentation. Neuroimage 2005;26:839-851.

20 Ashburner J, Friston KJ: Why voxel-based morphometry should be used. Neuroimage 2001;14:1238-1243.

-21 Whitwell JL, Jack CR Jr, Baker M, Rademakers R, Adamson J, Boeve BF, Knopman DS, Parisi JF, Petersen RC, Dickson DW, Hutton ML, Josephs KA: Voxel-based morphometry in frontotemporal lobar degeneration with ubiquitin-positive inclusions with and without progranulin mutations. Arch Neurol 2007;64:371-376.

-22 Amunts K, Kedo O, Kindler M, Pieperhoff P, Mohlberg H, Shah NJ, Habel U, Schneider F, Zilles K: Cytoarchitectonic mapping of the human amygdala, hippocampal region and entorhinal cortex: intersubject variability and probability maps. Anat Embryol (Berl) 2005;210:343-352.

-23 Espay AJ, Litvan I: Parkinsonism and frontotemporal dementia: the clinical overlap. J Mol Neurosci 2011;45: 343-349.

24 Boeve BF, Hutton M: Refining frontotemporal dementia with parkinsonism linked to chromosome 17: introducing FTDP-17 (MAPT) and FTDP-17 (PGRN). Arch Neurol 2008;65:460-464.

-25 Mackenzie IR, Baborie A, Pickering-Brown S, du Plessis D, Jaros E, Perry RH, Neary D, Snowden JS, Mann DM: Heterogeneity of ubiquitin pathology in frontotemporal lobar degeneration: classification and relation to clinical phenotype. Acta Neuropathol 2006;112:539-549.

-26 Mackenzie IR: The neuropathology and clinical phenotype of FTD with progranulin mutations. Acta Neuropathol 2007;114:49-54.

27 Sampathu DM, Neumann M, Kwong LK, et al: Pathological heterogeneity of frontotemporal lobar degeneration with ubiquitin-positive inclusions delineated by ubiquitin immunohistochemistry and novel monoclonal antibodies. Am J Pathol 2006;169:1343-1352.

-28 Mackenzie IR, Baker M, West G, et al: A family with tau-negative frontotemporal dementia and neuronal intranuclear inclusions linked to chromosome 17. Brain 2006;129:853-867.

29 Whitwell JL, Jack CR Jr, Parisi JE, Knopman DS, Boeve BF, Petersen RC, Dickson DW, Josephs KA: Imaging signatures of molecular pathology in behavioral variant frontotemporal dementia. J Mol Neurosci 2011;45:372378.

-30 Premi E, Grassi M, Gazzina S, Paghera B, Pepe D, Archetti S, Padovani A, Borroni B: The neuroimaging signature of frontotemporal lobar degeneration associated with granulin mutations: an effective connectivity study. J Nucl Med 2013;54:1066-1071.

-31 Rohrer JD, Warren JD, Omar R, Mead S, Beck J, Revesz T, Holton J, Stevens JM, Al-Sarraj S, Pickering-Brown SM, Hardy J, Fox NC, Collinge J, Warrington EK, Rossor MN: Parietal lobe deficits in frontotemporal lobar degeneration caused by a mutation in the progranulin gene. Arch Neurol 2008;65:506-513. 